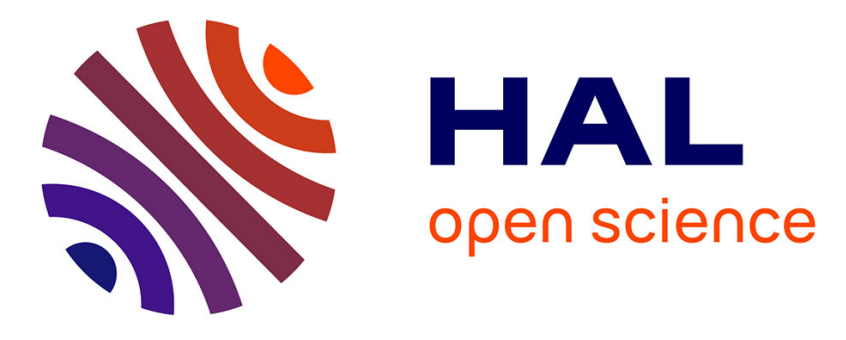

\title{
Imagined paralysis alters somatosensory evoked-potentials
}

\author{
Estelle Palluel, Caroline J Falconer, Christophe Lopez, Silvia Marchesotti, \\ Matthias Hartmann, Olaf Blanke, Fred W Mast
}

\section{- To cite this version:}

Estelle Palluel, Caroline J Falconer, Christophe Lopez, Silvia Marchesotti, Matthias Hartmann, et al. Imagined paralysis alters somatosensory evoked-potentials. Cognitive Neuroscience, 2020, 11 (4), pp.2015-215. 10.1080/17588928.2020.1772737 . hal-03009028

\section{HAL Id: hal-03009028 https://hal.science/hal-03009028}

Submitted on 17 Nov 2020

HAL is a multi-disciplinary open access archive for the deposit and dissemination of scientific research documents, whether they are published or not. The documents may come from teaching and research institutions in France or abroad, or from public or private research centers.
L'archive ouverte pluridisciplinaire HAL, est destinée au dépôt et à la diffusion de documents scientifiques de niveau recherche, publiés ou non, émanant des établissements d'enseignement et de recherche français ou étrangers, des laboratoires publics ou privés. 


\section{Imagined paralysis alters somatosensory evoked-potentials}

2

3 Estelle Palluel ${ }^{1,2}$, Caroline J. Falconer ${ }^{3,4}$, Christophe Lopez ${ }^{5}$, Silvia Marchesotti ${ }^{1}$, Matthias 4 Hartmann $^{3,6}$, Olaf Blanke $e^{1,7}$ and Fred W. Mast ${ }^{3}$

5

$6{ }^{1}$ Laboratory of Cognitive Neuroscience, Center for Neuroprosthetics and Brain Mind Institute, Swiss 7 Federal Institute of Technology (EPFL), 9 Chemin des Mines, 1202, Geneva, Switzerland

$8{ }^{2}$ Univ. Grenoble Alpes, CNRS, TIMC-IMAG, 38000 Grenoble, France.

$9{ }^{3}$ Department of Psychology, University of Bern, Bern, Switzerland

$10{ }^{4}$ Department of Psychiatry and Applied Psychology, University of Nottingham, Nottingham, U.K.

$11{ }^{5}$ Aix Marseille Univ, CNRS, LNSC, FR3C, Marseille, France

$12{ }^{6}$ Faculty of Psychology, Swiss Distance University Institute, Brig, Switzerland

$13 \quad{ }^{7}$ Department of Clinical Neurosciences, University Hospital Geneva, Geneva, Switzerland

14

15

16

17

18

19

20

21

22

Correspondence to:

Dr. Estelle Palluel

Laboratoire TIMC-IMAG, Team Santé-Plasticité-Motricité, UMR UJF-CNRS 5525, La Tronche, France.

Email: Estelle.palluel@univ-grenoble-alpes.fr 
29 Recent studies employing body illusions have shown that multisensory conflict can alter body representations and modulate low-level sensory processing. One defining feature of these body illusions is that they are sensory driven and thus passive on behalf of the participant. Thus, it remained to establish whether explicit alteration of own-body representations modulates low-level sensory processing. We investigated whether tibial nerve somatosensory-evoked potentials were modulated when participants imagined paralysis of their legs and arms. Imagined paralysis of the legs decreased P40 amplitude, but not imagined paralysis of the arms. These results show modulation of early somatosensory processing via explicit, top-down alteration to the internal representation of the body. Interestingly, P40 suppression positively correlated with bodily awareness scores whereas it negatively correlated with body dissociation scores. This suggests that the ability to actively alter own-body representation and its corresponding sensory processing depends upon dispositions to attend to and focus on bodily sensations.

41

42 Key words: Imagined paralysis, body representation, imagery, somatosensory-evoked potentials, 


\section{Introduction}

In the past decade, several studies have demonstrated the ability to modulate the perception of the body by multisensory conflicts in healthy participants (for a review see Blanke, 2012). For example, during the rubber hand illusion participants observe a rubber or virtual hand being stroked in front of them, while simultaneously experiencing their occluded real hand being stroked (Botvinick \& Cohen, 1998; Hara et al., 2015; Tsakiris \& Haggard, 2005). The integration of the simultaneous seen and felt stroking results in the participant feeling like the rubber hand is their hand, which is also accompanied by a drift in the perceived location of their real hand towards the rubber hand. This illusion has evolved to incorporate a whole virtual body, creating a full-body illusion, characterized by illusory self-identification with, and self-relocation towards, a virtual body (Lenggenhager, Tadi, Metzinger, \& Blanke, 2007).

Illusory self-identification with a fake/virtual body is associated with physiological changes, such as decreased skin temperature (Moseley et al., 2008; Salomon, Lim, Pfeiffer, Gassert, \& Blanke, 2013). However De Hann et al. (2017) and Nakul et al. (2020) showed that these results were not consistent. In addition, illusory self-identification with a body increased pain threshold in healthy participants (Hansel, Lenggenhager, Kanel, Curatolo, \& Blanke, 2011) and evoked mild analgesia in patients with spinal cord injury (Pozeg et al., 2017). Other studies have provided evidence that the full-body illusion modulated somatosensory information processing. Aspell, Palluel, \& Blanke (2012) have shown an increase in the amplitude of early (i.e. $40 \mathrm{~ms}$ post-stimulus onset) tibial nerve somatosensory evoked-potentials (SEPs) during the full-body illusion. Similarly, Dieguez and colleagues (2009) found that median nerve SEPs were larger during experimentally-induced illusion of numbness in the index finger. These results suggest a link between experimentally-induced changes in body representation and the way in which the brain processes sensory information, which also has direct physiological consequences in the case of skin temperature. One common aspect of these illusions is that they alter body representations through multisensory conflicts, which is passive on behalf of the participant. That is, participants are not engaged in a cognitive process in order to alter own-body representations. In the present study we investigated whether mental imagery of altered 
body representation is enough to modulate sensory processing in the brain, as previously shown during experimentally-induced alterations of body representation.

Imagined paralysis is a useful laboratory paradigm to alter own-body representations by mental imagery. Hartmann, Falconer, \& Mast (2011) instructed participants to imagine that they were paralysed from the waist down while simultaneously performing a mental imagery task (Parsons, 1987). This task requires that participants mentally align and transform the internal representation of their body composition (body representation) to match a target composition. Imagined leg paralysis impaired the participant's ability to mentally transform their body. This suggests that the biomechanical constraints associated with paralysis are incorporated into the body representation, impinging their ability to mentally transform it into another composition. The majority of research in this field has used mental transformations and motor imagery, which focus on the use of the body representation in performing simulated actions (Kakigi et al., 1997). Our paradigm allows to investigate the extent to which we can explicitly access and alter the body representation, and consequently somatosensory cortex activity.

The aim of the current study was to build upon the behavioural results of Hartmann, Falconer, \& Mast (2011) by investigating whether the top-down process of imagining leg paralysis can influence physiological signals relevant to the body representation. We hypothesize that imagined paralysis of the legs would influence the processing of tibial nerve signals in the somatosensory cortex. This is in contrast to imagined paralysis of the arms, which has no link with the tibial nerve. We used electroencephalography (EEG) recordings during tibial nerve electrical stimulation to assess SEP modulation during imagined paralysis. Our hypothesis is two-tailed in that there is evidence to suggest either an attenuation or amplification of SEPs and an altered body representation. On the one hand, evidence from conversion paralysis, a psychogenic inability to perform voluntary movement, shows a suppression of SEPs during the symptomatic period (Vuilleumier, 2005; Yazici, Demirci, Demir, \& Ertugrul, 2004). Furthermore, during hypnotic suggestion, imagery of the self-floating outside the body suppresses SEPs (V. De Pascalis, Magurano, Bellusci, \& Chen, 2001). On the other hand, alterations in body representation in the full-body illusion and numbness illusion have shown an 
amplification of SEPs (Aspell et al., 2012; Dieguez et al., 2009), in line with the deafferentation literature showing that a reduction in dominant sensory inputs allows for the expression of latent inputs (Tinazzi, Rosso, Zanette, Fiaschi, \& Aglioti, 2003; Urasaki, Genmoto, Wada, Yokota, \& Akamatsu, 2002).

\section{Participants}

Fourteen right-handed healthy volunteers took part (six females, eight males, age range: $22-$ 30 years). All participants gave written informed consent and were given monetary compensation for their participation. The study was approved by the local research ethics committee at École Polytechnique Fédérale de Lausanne. Data from four participants had to be discarded because they did not show identifiable SEP (SEP amplitudes have a large inter-individual variability: Aspell et al., 2012; Van de Wassenberg, Kruizinga, Van der Hoeven, Leenders, \& Maurits, 2008), thus ten participants data was included in the analysis (five females, five males, age range: $22-30$ years).

\section{Task and Procedures}

The experiment consisted of three blocks: Imagined Arm Paralysis, Imagined Leg Paralysis and Baseline. Each block included 1) an immersion task to induce the corresponding paralysis state or the baseline, 2) EEG recordings, and 3) two questionnaires to fill in at the end of each block. The immersion tasks lasted 2-5 minutes as each participant worked at their own pace and were to provide participants with an opportunity to draw upon an experience that emphasized the "uselessness" of either their arms or legs and therefore help to put themselves into the situation of being unable to move the respective body part. All participants remarked that the immersion tasks for the leg and arm paralysis were "challenging", "tricky" or said that "I didn't think it would be that difficult to do. For the arm paralysis, participants were imagining arm paralysis and were required to write "my name is..." using a pen inserted into the mouth during the immersion task. During EEG recordings they sat 
on a normal chair and were asked to imagine that both of their arms were paralyzed. They were instructed to imagine that they could not move nor feel their arms. For the leg paralysis, participants were required to navigate the experimental room in a manual wheelchair following an L-path during the immersion task. This was done twice and involved turning. During EEG recordings, they were asked to imagine that both of their legs were paralysed. They were instructed to imagine that they could not move nor feel their legs from the waist down. They remained seated on a manual wheelchair throughout the recordings in order to help to maintain the imagery state. For the baseline, participants were asked to walk twice following the same L-path than during the immersion task of the imagined leg paralysis. During EEG recordings, they sat relaxed in a normal chair without performing any task and with their arms on their legs. In all conditions participants were asked to keep their eyes closed. The three EEG recordings lasted 3.5 minutes each and were counterbalanced across participants. After each imagery block, participants answered two questionnaires pertaining to the perception of their leg and arm during imagined paralysis.

\section{Tibial Nerve Stimulation}

Participants received tibial nerve stimulation via two skin electrodes attached to the inside site of the right leg ankle. We used a Grass S48 stimulator to generate electrical pulses and a Matlab (version R2012B, MathWorks ${ }^{\circledR}$ ) script synchronized EEG recordings and electrical stimulation. During each experimental condition the tibial nerve was stimulated 400 times at a frequency of $2 \mathrm{~Hz}$ with pulse duration of $0.2 \mathrm{~ms}$ (for a similar procedure see Aspell et al., 2012) with intensity just below motor threshold (Hume \& Cant, 1978). No participant reported pain or discomfort with this level of stimulation.

\section{Electroencephalography: Acquisition and Pre-processing}

Continuous EEG was recorded with a sampling frequency of $2048 \mathrm{~Hz}$ from 64 active scalp electrodes (BioSemi, Netherlands) arranged in accordance with the 10-20 system and referenced to the common mode sense-driven right leg ground (CMS-DRL). Electrooculogram was recorded to control for artefacts related to eye movements, using a bipolar montage with electrodes positioned on 
the left and right side of the eyes (for horizontal eye movements), as well as above and below the right eye (for vertical movements). Participants were instructed to avoid to move their eyes.

EEG pre-processing and analysis were performed with the software Cartool version 3.8 (https://sites.google.com/site/cartoolcommunity/). EEG epochs were calculated from $100 \mathrm{~ms}$ before to $600 \mathrm{~ms}$ after the onset of right tibial nerve stimulation. A baseline correction was applied from 100 to $20 \mathrm{~ms}$ before stimulus onset. An artefact rejection threshold of $\pm 50 \mu \mathrm{V}$ was applied to both EEG and electrooculography signals. All accepted epochs were visually inspected and transient contaminating noise such as eye movements and muscle artefacts were rejected. On average 80 epochs $(20 \%)$ per participant were rejected. The data was band-pass filtered (1-40 Hz) with a $50 \mathrm{~Hz}$ notch filter.

\section{EEG Analysis}

Somatosensory-evoked potentials: Because tibial SEPs are maximal for midline electrodes $\mathrm{Cz}$ and $\mathrm{CPz}$ (Cruse, Klem, Lesser, \& Leuders, 1982), we defined ten target electrodes (Pz, CPz, CP1, $\mathrm{CP} 2, \mathrm{Cz}, \mathrm{C} 1, \mathrm{C} 2, \mathrm{FCz}, \mathrm{FC} 1, \mathrm{FC} 2)$ located in a region of interest around $\mathrm{Cz}$ and $\mathrm{CPz}$. Individual SEPs were calculated for each target electrode and each condition after normalization to their mean global field power (GFP). GFP is the spatial standard deviation of the scalp's electrical field at a given moment in time (Mercier, Schwartz, Michel, \& Blanke, 2009) and normalization by the GFP reduces inter-subjects variability. Individual SEPs were then combined across participants to create grandaverage SEPs for each target electrode.

We looked for classical early tibial SEP components in each participant. The first cortical component of tibial stimulation appears ipsilateral to the leg of stimulation at $40 \mathrm{~ms}$ (P40) (Cruse et al., 1982; Kakigi et al., 1995; Kakigi et al., 1997). Tibial SEP components also include the N50 ( 50 $\mathrm{ms})$ and P60 ( $\sim 60 \mathrm{~ms})$, all of which originate from area $3 \mathrm{~b}$ in the primary somatosensory cortex (Kakigi et al., 1995). Accordingly, three time windows corresponding to each of the SEP components were considered: $30-45 \mathrm{~ms}, 45-60 \mathrm{~ms}$ and $60-75 \mathrm{~ms}$. As the Shapiro-Wilk tests confirmed the normality of data, SEP amplitudes from each target electrode were subjected to a repeated-measures 
analysis of variance (ANOVA) with two factors: experimental condition (Baseline, Imagined Arm Paralysis, Imagined Leg Paralysis) and SEP component (P40, N50, P60).

Scalp topography: Topographical analysis of tibial SEP was conducted to investigate periods of stable brain activation within and between experimental conditions (Murray, Brunet, \& Michel, 2008). EP topographical analysis searches for time-segments of stable map topography that represent functional microstates of the brain during information processing. This method has been applied to the analysis of tibial SEPs (Aspell et al., 2012), visual EPs (Lopez, Mercier, Halje, \& Blanke, 2011), and auditory Eps (Giroud, Lemke, Reich, Matthes, \& Meyer, 2017). In the first step of the analysis, EP topographies (maps or microstate segments) were established using a spatial clustering algorithm (Tibshirani \& Walther, 2005). The cluster analysis is dependent upon the global explained variance (GEV), which is the goodness of fit to a template map during a specific time period and the strength of the global field power (GFP) during that time period (Murray et al., 2008). This step identifies the dominant map topographies on the scalp in the group-average SEP data during and across the three experiment conditions. In the second step, we analysed the extent to which these dominant maps are present and verified in participant data. This was achieved by performing a fitting procedure based on the spatial correlations between participant data and the template maps identified from the groupaverage EP data in the temporal domain (Lopez et al., 2011). Participant data corresponding to the dominant maps established in the first step are subjected to statistical analysis (two-tailed $t$-test). In keeping with our investigation into somatosensory processing, we analysed map topographies only during the time windows that correspond to SEPs.

\section{Questionnaires}

After each imagined paralysis condition, participants completed two questionnaires concerned with the perception of their legs and arms during imagery (adapted from Hartmann et al., 2011). Participants rated on a 7-point Likert scale $(1=$ not true, $7=$ true $)$ the extent to which they agreed with seven statements (Table 1). As in Hartman et al. (2011) we averaged the seven statements concerned with the perception of the legs for each participant and for each state of paralysis. The 
seven statements concerned with the perception of the arms were also averaged for each participant and for each state of paralysis. The averaged scores were compared for both conditions by means of non-parametric Friedman ANOVA and Wilcoxon. We compared ratings for leg-related statement during imagined paralysis of the legs and imagined paralysis of the arms. This was also done for armrelated statements. Bonferroni correction was applied in order to account for multiple comparisons.

At the end of the experiment participants completed two questionnaires. The Private Body Consciousness Subscale (PBCS; Miller, Murphy, \& Buss, 1981) is a 5-item questionnaire gauging the extent to which participants are consciously aware of internal bodily processes. Participants rated on a 5-point scale $(1=$ not true of me, 5 = very true of me $)$ the extent to which they agreed with statements such as "I am sensitive to internal bodily tensions". The Scale of Body Connection (SBC; Price \& Thompson, 2007) gauge the extent to which participants can connect with or disconnect from bodily processes (for a review see Mehling et al., 2009). Participants rated on a 5-point scale ( $1=$ not at all, 5 = all the time) the extent to which they agreed with statements pertaining to two subscales measuring body awareness (12 items, e.g., "Take cues from the body") and body dissociation (8 items, e.g., "Feel separated from body"). Average scores from each of the scales were correlated with the maximum amplitude difference between the imagined leg paralysis and the Baseline condition using Spearman correlations. Statistical analysis was performed using Statistica 10.

\section{EEG Results}

\section{$\underline{\text { Results }}$}

Somatosensory-evoked potentials: As predicted, the results show a significant modulation of the SEP P40 component during imagined leg paralysis, but not during imagined arm paralysis, when compared to Baseline conditions.

The ANOVA revealed a significant main effect of SEP component for each target electrode, whereby the N50 amplitude was significantly lower than the P40 and P60 amplitudes (all $F(2,18)>$ 4.2 and $p<0.032, \eta_{\mathrm{p}}{ }^{2}=0.32$ ). None of the target electrodes showed a significant main effect of the paralysis condition $(p>0.05)$. Interestingly, electrode $\mathrm{CPz}$ showed a significant interaction between 
the paralysis condition and the SEP component $\left(F(4,36)=3.49, p=0.016, \eta_{p}{ }^{2}=0.28\right)$ (Fig. 1). Tukey post-hoc tests revealed that the P40 amplitude (mean $\pm \mathrm{SEM}, 0.51 \mu \mathrm{V} \pm 0.16 \mu \mathrm{V}$ ) was lower during Imagined Leg Paralysis when compared to the Baseline condition $(0.90 \mu \mathrm{V} \pm 0.16 \mu \mathrm{V}, p=0.033)$. Yet the amplitude did not differ from the Imagined Arm Paralysis condition $(0.83 \mu \mathrm{V} \pm 0.28 \mu \mathrm{V}, p=0.25)$ (Fig. 2). The analysis also revealed a trend for a similar interaction at electrodes $\mathrm{Cz}(F(4,36)=2.39, p$ $\left.=0.069, \eta_{p}{ }^{2}=0.210\right)$ and $\mathrm{C} 2\left(F(4,36)=2.47, p=0.062, \eta_{p}{ }^{2}=0.216\right)$.

\section{Please insert figures 1 and 2 about here}

Scalp topography: Analysis of group-average EPs revealed eight sequential maps of stable brain activation (up to $175 \mathrm{~ms}$ after stimulus onset) following tibial stimulation in all three conditions (Fig. 3). We analysed Map 4 ( 25-60 ms) and Map 5 ( $\sim 60-90 \mathrm{~ms})$ overlapping with the main SEP components (30-75ms). The maximum GFP for Map 4 was significantly reduced for Imagined Leg Paralysis (mean $\pm \mathrm{SEM}, 0.55 \mu \mathrm{V} \pm 0.09 \mu \mathrm{V})$ when compared to the Baseline condition $(0.73 \mu \mathrm{V} \pm$ $0.10 \mu \mathrm{V} ; t(9)=2.38, p=0.041, \mathrm{~d}=0.71)$. No statistically significant differences were found between the Baseline and Imagined Arm Paralysis conditions $(t(9)=0.92, p=0.382, \mathrm{~d}=0.25$; Figure 4). Interestingly, the P40 component falls within Map 4 and the maximum positivity in Map 4 is at electrode CPz. There was no significant difference between the arm and leg paralysis conditions for Map 5.

\section{Please insert figures 3 and 4 about here}

\section{Questionnaires}

Limb perception: Participants reported perceptual changes in the limbs congruent with the imagined paralysis condition (Table 1). A non-parametric Friedman ANOVA was conducted and rendered a Chi-square value of 25.2, which was significant $(p<0.001)$. Participants rated the legs statement significantly higher after imagined leg paralysis than imagined arm paralysis $(z=2.8 ; p=$ $0.005, \mathrm{~d}=0.83)$. The reverse was true for arm-related statements during imagined arm paralysis $(z=$ 
2.8; $p=0.005, \mathrm{~d}=0.64)$. A non-parametric Friedman ANOVA was conducted and rendered a Chisquare value of 25.2, which was significant $(p<0.001)$. We used Bonferroni correction for multiple comparisons; $\alpha$ corr $=0.05 / 4=0.013$, and the effect reported above was still statistically significant. There was no significant difference between the rating of arm statements during imagined arm paralysis and leg statements during imagined leg paralysis $(z=1.22, p=0.221, \mathrm{~d}=0.59)$. The intensity of perceptual changes between arms and legs during their corresponding paralysis conditions were not significantly different. In conclusion, subjective perception of the limbs was differentially influenced by the two paralysis conditions (Figure 5).

Bodily consciousness: Spearman correlations revealed a significant negative correlation between maximum peak amplitude difference of the P40 between the Baseline and imagined leg paralysis conditions and $\operatorname{PBCS}$ scores $(\rho=-0.78, p=0.008)$. That is, those scoring high in their awareness of body sensations, exhibit the greatest amount of amplitude suppression of the P40 component during imagined leg paralysis (Figure 5). Conversely, those scoring high on the body dissociation (BD) subscale of the SBC showed the least amount P40 amplitude suppression during imagined leg paralysis $(\rho=0.65, p=0.028)$. That is, participants who have a higher disposition to disconnect themselves from sensory and emotional states show less modulation of somatosensory processing. By contrast, the body awareness subscale of the SBC did not correlate significantly with SEP data $(\rho=-0.28, p=0.441)$.

\section{Please insert figures 5 and 6 about here}

\section{$\underline{\text { Discussion }}$}

We examined whether imagined paralysis modulates somatosensory information processing. We found modulation of tibial nerve SEPs compared to a Baseline condition, specifically during imagined paralysis of the legs, but not of the arms. This effect is characterised by a reduction in the maximum peak amplitude of the $\mathrm{P} 40$ at electrode $\mathrm{CPz}$, suggesting that imagined paralysis modulates sensory processing of the leg in primary somatosensory cortex (S1). In addition to single trace 
analysis, we showed that imagined paralysis influenced topographical brain, whereby the GFP decreased during imagined paralysis of the legs, but not of the arms, when compared to a Baseline condition. GFP modulation corresponds to a topographical map overlapping with the P40 SEP component, thus corroborating findings from single trace analysis.

We have previously shown that imagined paralysis can influence mental imagery of the body (Hartmann et al., 2011). During imagined paralysis, the biomechanical constraints of paralysis are attributed to the limbs represented in the body representation, impinging upon the ability to mentally transform them. Subjective reports indicate that imagined paralysis evoked weakness, heaviness, and immobility of the limbs (Hartmann et al., 2011). Here, we expand upon our understanding of paralysis imagery by showing that it is a cognitive process which is able to modulate low-level somatosensory information processing.

Modified somatosensory processing has previously been shown during experimentallyinduced body illusions (Aspell et al., 2012; Dieguez et al., 2009). The SEP amplification found in these studies is consistent with SEP amplitude increases in response to transient physical anaesthesia of a body part (Tinazzi et al., 2003; Urasaki et al., 2002). The increase in SEP amplitude accompanying altered body representation found by Aspell et al. (2012) and Dieguez et al. (2009) is likely the consequence of multisensory reweighting of signals in the brain. In contrast with these findings, our results showed P40 suppression during imagined paralysis. One of the major differences between altered body representation achieved in these studies and in the current study is that Aspell et al. (2012) and Dieguez et al. (2009) altered body representations via spatiotemporal multisensory conflicts. Although participants consciously perceived altered body representations in these studies, it was somewhat sensory driven and did not require active cognitive effort on their behalf. Paralysis imagery, on the other hand, requires participants to consciously and mentally act to alter own-body representations. Thus, attenuation of SEPs in the present study may reflect explicit efforts to alter the body representation and inhibit sensory perceptions of the legs that might not be considered as crucial by the central nervous system. Our results are consistent with several studies using hypnotic suggestion. For example, De Pascalis, Cacace, \& Massicolle (2008) instructed participants to imagine 
an obstructive object (a glove) between their hand and a painful stimulus, and found a reduction in the N140 and P200 amplitudes. Likewise, De Pascalis et al. (2008) found a similar suppression of the N140 and P200 responses to painful stimulation when participants imagined floating outside of their body. While the body representation was not the primary target of these studies, they suggest that own-body imagery can modulate somatosensory processing.

Later activation changes have also been observed in the literature. Long-latency ( $>40 \mathrm{~ms})$ components are thought to be generated by several areas, including area $3 \mathrm{~b}$, areas 1 and 2 , secondary somatosensory cortex (SII), and primary motor cortex (area 4). SEP components to tibial nerve stimulation around the time period of the later response at $110-200 \mathrm{~ms}$ originate in or near to SII, as well as in the posterior parietal and frontal cortex (Allison, McCarthy, Wood, Darcey, et al., 1989; Allison, McCarthy, Wood, Williamson, \& Spencer, 1989; Kakigi et al., 1995). These later changes were mostly attributed to the detection of visuo-tactile conflicts that were not present in our study (Aspell et al., 2012; Press, Heyes, Haggard, \& Eimer, 2008). Heydrich et al. (2018) suggested that areas beyond S1 (such as the secondary motor cortex, see Bufalari, Aprile, Avenanti, Di Russo, \& Aglioti, 2007) are associated with changes in somatosensory processing linked to altered states of bodily self-consciousness (e.g. illusory self-identification with an avatar). However the electrophysiological data of Press et al. (2008) indicate that the process of filtering what may or may not become part of one's body is not the same as volitional alteration of body representation.

Another interesting result of the present study was that the magnitude of SEP suppression correlated with PBCS and body dissociation scores. Those with the disposition to attend to and focus on bodily sensations exhibited the largest SEP suppression. It is reasonable to assume that heightened bodily awareness results in increased accessibility to the internal representation of the body and an enhanced ability to impose upon this sensory representation. Conversely, those scoring high in body dissociation exhibited the least amount of suppression, or showed the reverse and exhibited an increase in SEP amplitudes. It could be the case that those exhibiting high body dissociation are using a less sensory-bound body representation to achieve imagined paralysis. However, further research 
should aim to elaborate on these findings and establish the causal role of body awareness and body dissociation in accessing and manipulating own-body representations.

While the underlying mechanisms of top-down modulation in S1 remain a topic for future research, our findings still have a significant clinical implication for Mindfulness Based Therapy (MBT) and motor imagery (MI) in the presence of pain. Recent trends in clinical intervention have ventured towards MBT (Baer, 2003), an intervention that encourages patients to focus on their current internal bodily sensations, emotions and thoughts with an attitude of non-judgmental acceptance. MBT has been successful in the treatment of acute anxiety and depression (Hofmann, Sawyer, Witt, $\&$ Oh, 2010), as well as in reducing stress and symptoms associated with physical and psychosomatic disorders (Grossman, Niemann, Schmidt, \& Walach, 2004), including pain (Rosenzweig et al., 2010) and irritable bowel syndrome (Ljotsson et al., 2010; Zernicke et al., 2013). One commonality between MBT and paralysis imagery is that they shift attention towards a specific body part and require participants to focus on the sensory perception of that body part. In light of our findings, future research should establish whether MBT similarly influences low-level sensory processing. Such evidence would enable MBT to be tailored to patients with specific body related disorders. Modifying the body schema via motor imagery (MI) could also be a promising approach for the treatment of clinical disorders. MI refers to the mental representation of an action without engaging in its actual execution (Moran, Guillot, Macintyre, \& Collet, 2012). It involves the absence of overt motor output rather than of overt movement itself (MacIntyre, Madan, Moran, Collet, \& Guillot, 2018). The repetitive use of MI has been shown to promote motor recovery and to alleviate phantom-limb pain in lower limb amputees (Saruco et al., 2019). However, Gustin et al. (2008) reported exacerbation of pain in response to MI in spinal cord injury patients with neuropathic pain. These authors suggested that "the generation of pain may be an example of a "mental" or "cognitive" allodynia where activation of sensory pathways on a background of central neuronal hyperexcitability or sensitization results in the generation of pain". Thus, imagined paralysis or the "no movement" imagery may have analgesic effects in patients with chronic arm/leg pain or phantom limb pain, for example. Further research should be conducted as MI is altered in a number of pathologies characterized by an 
impairment of action performances (Coslett, Medina, Kliot, \& Burkey, 2010; Fiori et al., 2013; Ionta et al., 2016; Scandola, Aglioti, Avesani, et al., 2017; Scandola, Aglioti, Pozeg, Avesani, \& Moro, 2017; Schwoebel, Friedman, Duda, \& Coslett, 2001).

A limitation of the current study is the relatively low sample size, which can result in an overestimation of the observed effect. A replication of our findings in a higher-powered sample is therefore desirable to test the robustness of this effect. To conclude, we have confirmed that the body representation is malleable via conscious, top-down processes. Previous research has also shown that body representation can be influenced by passive manipulations to multisensory signals, resulting in altered low-level sensory processing. The current study expands upon these results by showing that low-level sensory processing can be modulated by explicit, top-down alterations to the body representation. Paralysis imagery can penetrate early levels of somatosensory processing.

\section{$\underline{\text { Author contributions }}$}

E. Palluel and C.J. Falconer developed the study concept. All authors contributed to the study design. Testing and data collection were performed by E. Palluel and C.J. Falconer. E. Palluel, C.J. Falconer, C. Lopez, S. Marchesotti, M. Hartman performed the data analysis and interpretation under the supervision of O. Blanke and F.W. Mast. E. Palluel and C.J. Falconer drafted the manuscript, and C. Lopez and M. Hartman provided critical revisions. All authors approved the final version of the manuscript for submission.

\section{Disclosure Statement}

The authors report no conflict of interest 
Allison, T., McCarthy, G., Wood, C. C., Darcey, T. M., Spencer, D. D., \& Williamson, P. D. (1989). Human cortical potentials evoked by stimulation of the median nerve. I. Cytoarchitectonic areas generating short-latency activity. J Neurophysiol, 62(3), 694-710. doi:10.1152/jn.1989.62.3.694

Allison, T., McCarthy, G., Wood, C. C., Williamson, P. D., \& Spencer, D. D. (1989). Human cortical potentials evoked by stimulation of the median nerve. II. Cytoarchitectonic areas generating long-latency activity. J Neurophysiol, 62(3), 711-722. doi:10.1152/jn.1989.62.3.711

Aspell, J. E., Palluel, E., \& Blanke, O. (2012). Early and late activity in somatosensory cortex reflects changes in bodily self-consciousness: an evoked potential study. Neuroscience, 216, 110-122. doi:10.1016/j.neuroscience.2012.04.039

Baer, R. A. (2003). Mindfulness Training as a Clinical Intervention: A Conceptual and Empirical Review. Clinical Psychology: Science and Practice, 10(2), 125-143. doi:10.1093/clipsy.bpg015

Blanke, O. (2012). Multisensory brain mechanisms of bodily self-consciousness. Nat Rev Neurosci, 13(8), 556-571. Retrieved from http://dx.doi.org/10.1038/nrn3292

Botvinick, M., \& Cohen, J. (1998). Rubber hands 'feel' touch that eyes see. Nature, 391(6669), 756. Retrieved

from http://www.ncbi.nlm.nih.gov/entrez/query.fcgi?cmd=Retrieve\&db=PubMed\&dopt=Citation \&list_uids $=9486643$

Bufalari, I., Aprile, T., Avenanti, A., Di Russo, F., \& Aglioti, S. M. (2007). Empathy for pain and touch in the human somatosensory cortex. Cereb Cortex, 17(11), 2553-2561. doi:10.1093/cercor/bhl161

Coslett, H. B., Medina, J., Kliot, D., \& Burkey, A. R. (2010). Mental motor imagery indexes pain: the hand laterality task. Eur J Pain, 14(10), 1007-1013. doi:10.1016/j.ejpain.2010.04.001

Cruse, R., Klem, G., Lesser, R. P., \& Leuders, H. (1982). Paradoxical lateralization of cortical potentials evoked by stimulation of posterior tibial nerve. Arch Neurol, 39(4), 222-225. doi:10.1001/archneur.1982.00510160028005

de Haan, A. M., Van Stralen, H. E., Smit, M., Keizer, A., Van der Stigchel, S., \& Dijkerman, H. C. (2017). No consistent cooling of the real hand in the rubber hand illusion. Acta Psychol (Amst), 179, 68-77. doi:10.1016/j.actpsy.2017.07.003

De Pascalis, V., Cacace, I., \& Massicolle, F. (2008). Focused analgesia in waking and hypnosis: Effects on pain, memory, and somatosensory event-related potentials. Pain, 134(1-2), 197-208. Retrieved from http://www.sciencedirect.com/science/article/pii/S0304395907005143

De Pascalis, V., Magurano, M. R., Bellusci, A., \& Chen, A. C. (2001). Somatosensory event-related potential and autonomic activity to varying pain reduction cognitive strategies in hypnosis. Clin Neurophysiol, 112(8), 1475-1485. doi:S1388245701005867 [pii]

Dieguez, S., Mercier, M. R., Newby, N., \& Blanke, O. (2009). Feeling numbness for someone else's finger. Curr Biol, 19(24), R1108-1109. doi:10.1016/j.cub.2009.10.055

Fiori, F., Sedda, A., Ferre, E. R., Toraldo, A., Querzola, M., Pasotti, F., . . Bottini, G. (2013). Exploring motor and visual imagery in Amyotrophic Lateral Sclerosis. Exp Brain Res, 226(4), 537-547. doi:10.1007/s00221-013-3465-9

Giroud, N., Lemke, U., Reich, P., Matthes, K. L., \& Meyer, M. (2017). The impact of hearing aids and age-related hearing loss on auditory plasticity across three months - An electrical neuroimaging study. Hear Res, 353, 162-175. doi:10.1016/j.heares.2017.06.012

Grossman, P., Niemann, L., Schmidt, S., \& Walach, H. (2004). Mindfulness-based stress reduction and health benefits: A meta-analysis. Journal of Psychosomatic Research, 57(1), 35-43. Retrieved from http://www.sciencedirect.com/science/article/pii/S0022399903005737

Gustin, S. M., Wrigley, P. J., Gandevia, S. C., Middleton, J. W., Henderson, L. A., \& Siddall, P. J. (2008). Movement imagery increases pain in people with neuropathic pain following complete thoracic spinal cord injury. Pain, 137(2), 237-244. doi:10.1016/j.pain.2007.08.032 
Hansel, A., Lenggenhager, B., Kanel, R. V., Curatolo, M., \& Blanke, O. (2011). Seeing and identifying with a virtual body decreases pain perception. Eur J Pain. doi:10.1016/j.ejpain.2011.03.013

Hara, M., Pozeg, P., Rognini, G., Higuchi, T., Fukuhara, K., Yamamoto, A., . . Salomon, R. (2015). Voluntary self-touch increases body ownership. Front Psychol, 6, 1509. doi:10.3389/fpsyg.2015.01509

Hartmann, M., Falconer, C. J., \& Mast, F. W. (2011). Imagined paralysis impairs embodied spatial transformations. Cogn Neurosci, 2(3-4), 155-162. doi:10.1080/17588928.2011.594498

Heydrich, L., Aspell, J. E., Marillier, G., Lavanchy, T., Herbelin, B., \& Blanke, O. (2018). Cardio-visual full body illusion alters bodily self-consciousness and tactile processing in somatosensory cortex. Sci Rep, 8(1), 9230. doi:10.1038/s41598-018-27698-2

Hofmann, S. G., Sawyer, A. T., Witt, A. A., \& Oh, D. (2010). The effect of mindfulness-based therapy on anxiety and depression: A meta-analytic review. J Consult Clin Psychol, 78(2), 169-183. doi:10.1037/a0018555

Hume, A. L., \& Cant, B. R. (1978). Conduction time in central somatosensory pathways in man. The averaged scores were compared for both conditions by means of non-parametric Friedman ANOVA and Wilcoxon. Spinal cord injury affects the interplay between visual and sensorimotor representations of the body. Sci Rep, 6, 20144. doi:10.1038/srep20144

Kakigi, R., Koyama, S., Hoshiyama, M., Shimojo, M., Kitamura, Y., \& Watanabe, S. (1995). Topography of somatosensory evoked magnetic fields following posterior tibial nerve stimulation. Electroencephalogr Clin Neurophysiol, 95(2), 127-134. doi:10.1016/0013-4694(95)00053-2

Kakigi, R., Shimojo, M., Hoshiyama, M., Koyama, S., Watanabe, S., Naka, D., . . Nakamura, A. (1997). Effects of movement and movement imagery on somatosensory evoked magnetic fields following posterior tibial nerve stimulation. Brain Res Cogn Brain Res, 5(3), 241-253. doi:S0926-6410(97)00002-5 [pii]

Lenggenhager, B., Tadi, T., Metzinger, T., \& Blanke, O. (2007). Video Ergo Sum: Manipulating Bodily Self-Consciousness. Science, 317(5841), 1096. Retrieved from http://science.sciencemag.org/content/317/5841/1096.abstract

Ljotsson, B., Andreewitch, S., Hedman, E., Ruck, C., Andersson, G., \& Lindefors, N. (2010). Exposure and mindfulness based therapy for irritable bowel syndrome--an open pilot study. J Behav Ther Exp Psychiatry, 41(3), 185-190. doi:10.1016/j.jbtep.2010.01.001

Lopez, C., Mercier, M. R., Halje, P., \& Blanke, O. (2011). Spatiotemporal dynamics of visual vertical judgments: early and late brain mechanisms as revealed by high-density electrical neuroimaging. Neuroscience, 181, 134-149. doi:10.1016/j.neuroscience.2011.02.009

Macintyre, T. E., Madan, C. R., Moran, A. P., Collet, C., \& Guillot, A. (2018). Motor imagery, performance and motor rehabilitation. Prog Brain Res, 240, 141-159. doi:10.1016/bs.pbr.2018.09.010

Mehling, W. E., Gopisetty, V., Daubenmier, J., Price, C. J., Hecht, F. M., \& Stewart, A. (2009). Body awareness: construct and self-report measures. PLOS ONE, 4(5), e5614. doi:10.1371/journal.pone.0005614

Mercier, M., Schwartz, S., Michel, C. M., \& Blanke, O. (2009). Motion direction tuning in human visual cortex. European Journal of Neuroscience, 29(2), 424-434. doi:10.1111/j.14609568.2008.06583.x

Miller, L. C., Murphy, R., \& Buss, A. H. (1981). Consciousness of Body - Private and Public. Journal of Personality and Social Psychology, 41(2), 397-406.

Moran, A., Guillot, A., Macintyre, T., \& Collet, C. (2012). Re-imagining motor imagery: building bridges between cognitive neuroscience and sport psychology. Br J Psychol, 103(2), 224-247. doi:10.1111/j.2044-8295.2011.02068.x

Moseley, G. L., Olthof, N., Venema, A., Don, S., Wijers, M., Gallace, A., \& Spence, C. (2008). Psychologically induced cooling of a specific body part caused by the illusory ownership of an artificial counterpart. Proc Natl Acad Sci U S A, 105(35), 13169-13173. doi:10.1073/pnas.0803768105 
Murray, M. M., Brunet, D., \& Michel, C. M. (2008). Topographic ERP analyses: a step-by-step tutorial review. Brain Topogr, 20(4), 249-264. doi:10.1007/s10548-008-0054-5

Nakul, E., Orlando-Dessaints, N., Lenggenhager, B., \& Lopez, C. (2020). Measuring perceived selflocation in virtual reality. Scientific reports, in press.

Parsons, L. M. (1987). Imagined spatial transformation of one's body. J Exp Psychol Gen, 116(2), 172191.

Retrieved from http://www.ncbi.nlm.nih.gov/entrez/query.fcgi?cmd=Retrieve\&db=PubMed\&dopt=Citation \&list_uids $=2955072$

Pozeg, P., Palluel, E., Ronchi, R., Solca, M., Al-Khodairy, A. W., Jordan, X., . . . Blanke, O. (2017). Virtual reality improves embodiment and neuropathic pain caused by spinal cord injury. Neurology, 89(18), 1894-1903. doi:10.1212/WNL.0000000000004585

Press, C., Heyes, C., Haggard, P., \& Eimer, M. (2008). Visuotactile learning and body representation: an ERP study with rubber hands and rubber objects. J Cogn Neurosci, 20(2), 312-323. doi:10.1162/jocn.2008.20022

Price, C. J., \& Thompson, E. A. (2007). Measuring dimensions of body connection: body awareness and bodily dissociation. J Altern Complement Med, 13(9), 945-953. doi:10.1089/acm.2007.0537

Rosenzweig, S., Greeson, J. M., Reibel, D. K., Green, J. S., Jasser, S. A., \& Beasley, D. (2010). Mindfulness-based stress reduction for chronic pain conditions: Variation in treatment outcomes and role of home meditation practice. Journal of Psychosomatic Research, 68(1), 29-36. Retrieved from http://www.sciencedirect.com/science/article/pii/S0022399909000944

Salomon, R., Lim, M., Pfeiffer, C., Gassert, R., \& Blanke, O. (2013). Full body illusion is associated with widespread skin temperature reduction. Front Behav Neurosci, 7, 65. doi:10.3389/fnbeh.2013.00065

Saruco, E., Guillot, A., Saimpont, A., Di Rienzo, F., Durand, A., Mercier, C., . . . Jackson, P. (2019). Motor imagery ability of patients with lower-limb amputation: exploring the course of rehabilitation effects. Eur J Phys Rehabil Med, 55(5), 634-645. doi:10.23736/S19739087.17.04776-1

Scandola, M., Aglioti, S. M., Avesani, R., Bertagnoni, G., Marangoni, A., \& Moro, V. (2017). Corporeal illusions in chronic spinal cord injuries. Conscious Cogn, 49, 278-290. doi:10.1016/j.concog.2017.01.010

Scandola, M., Aglioti, S. M., Pozeg, P., Avesani, R., \& Moro, V. (2017). Motor imagery in spinal cord injured people is modulated by somatotopic coding, perspective taking, and post-lesional chronic pain. J Neuropsychol, 11(3), 305-326. doi:10.1111/jnp.12098

Schwoebel, J., Friedman, R., Duda, N., \& Coslett, H. B. (2001). Pain and the body schema: evidence for peripheral effects on mental representations of movement. Brain, 124(Pt 10), 20982104. doi:10.1093/brain/124.10.2098

Tibshirani, R., \& Walther, G. (2005). Cluster Validation by Prediction Strength. Journal of Computational and Graphical Statistics, 14(3), 511-528. doi:10.1198/106186005×59243

Tinazzi, M., Rosso, T., Zanette, G., Fiaschi, A., \& Aglioti, S. M. (2003). Rapid modulation of cortical proprioceptive activity induced by transient cutaneous deafferentation: neurophysiological evidence of short-term plasticity across different somatosensory modalities in humans. Eur J Neurosci, 18(11), 3053-3060. doi:10.1111/j.1460-9568.2003.03043.x

Tsakiris, M., \& Haggard, P. (2005). The rubber hand illusion revisited: visuotactile integration and self-attribution. J Exp Psychol Hum Percept Perform, 31(1), 80-91. doi:2005-01366-006 [pii] 10.1037/0096-1523.31.1.80

Urasaki, E., Genmoto, T., Wada, S., Yokota, A., \& Akamatsu, N. (2002). Dynamic changes in area 1 somatosensory cortex during transient sensory deprivation: a preliminary study. J Clin Neurophysiol, 19(3), 219-231. Retrieved from http://www.ncbi.nlm.nih.gov/pubmed/12226567 
Van de Wassenberg, W. J., Kruizinga, W. J., Van der Hoeven, J. H., Leenders, K. L., \& Maurits, N. M. (2008). Multichannel recording of tibial-nerve somatosensory evoked potentials. Neurophysiol Clin, 38(5), 277-288. Retrieved from http://www.ncbi.nlm.nih.gov/entrez/query.fcgi?cmd=Retrieve\&db=PubMed\&dopt=Citation \&list_uids $=18940615$

Vuilleumier, P. (2005). Hysterical conversion and brain function. Prog Brain Res, 150, 309-329. doi:S0079-6123(05)50023-2 [pii]

Yazici, K. M., Demirci, M., Demir, B., \& Ertugrul, A. (2004). Abnormal somatosensory evoked potentials in two patients with conversion disorder. Psychiatry Clin Neurosci, 58(2), 222-225. Retrieved from http://www.ncbi.nlm.nih.gov/pubmed/15009831

Zernicke, K. A., Campbell, T. S., Blustein, P. K., Fung, T. S., Johnson, J. A., Bacon, S. L., \& Carlson, L. E. (2013). Mindfulness-based stress reduction for the treatment of irritable bowel syndrome symptoms: a randomized wait-list controlled trial. Int J Behav Med, 20(3), 385-396. doi:10.1007/s12529-012-9241-6 
During the experiment I had the impression that I couldn't use my legs as well as I am $1.00(0.00)$ used to.

During the experiment my legs felt weak

During the experiment my legs felt strange, as if they did not belong to my body

During the experiment I had the impression that I could not have moved my legs if I had wanted to

Arms

During the experiment, my arms felt useless

My arms felt heavy during the experiment

During the experiment I had the impression that I couldn't use my arms as well as I am used to.

During the experiment my arms felt weak

During the experiment my arms felt strange, as if they did not belong to my body

During the experiment my arms felt paralyzed

During the experiment I had the impression that I could not have moved my arms if I $2.77(0.83)$ had wanted to

$\begin{array}{ll}3.33(1.32) & 1.00(0.00) \\ 1.33(0.50) & 1.00(0.00) \\ 3.22(1.39) & 1.00(0.00) \\ 2.77(0.83) & 1.00(0.00)\end{array}$

Table 1. Mean and standard deviations in relation to seven statements concerned with the perception of either the legs or arms during paralysis imagery. Statements are rated on a 7point Likert scale $(1=$ not true, $7=$ true $)$. 


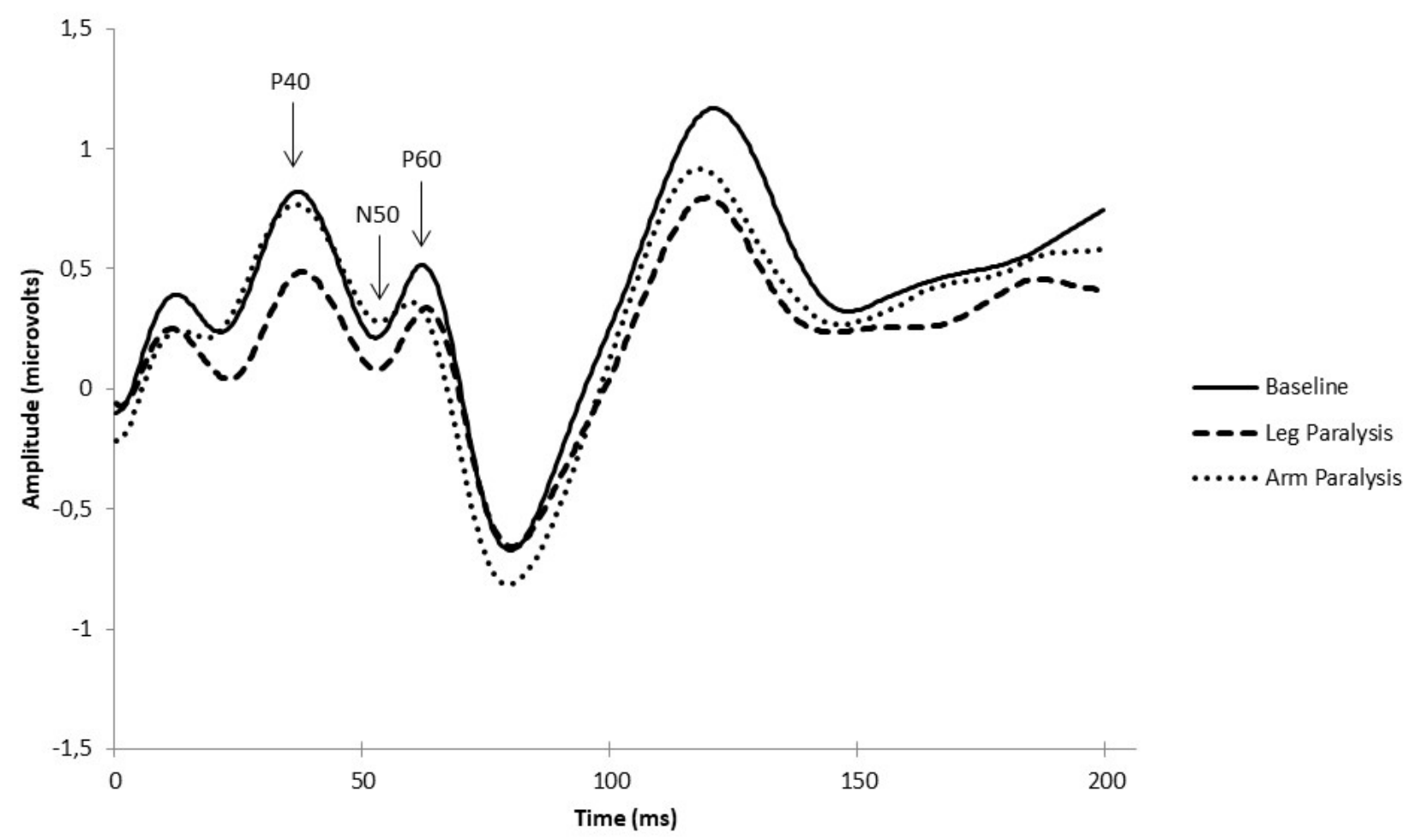

561

562 Figure 1. Single traces for electrode $\mathrm{CPz}$ depicting $\mathrm{SEP}$ components for all three 563 experimental conditions 


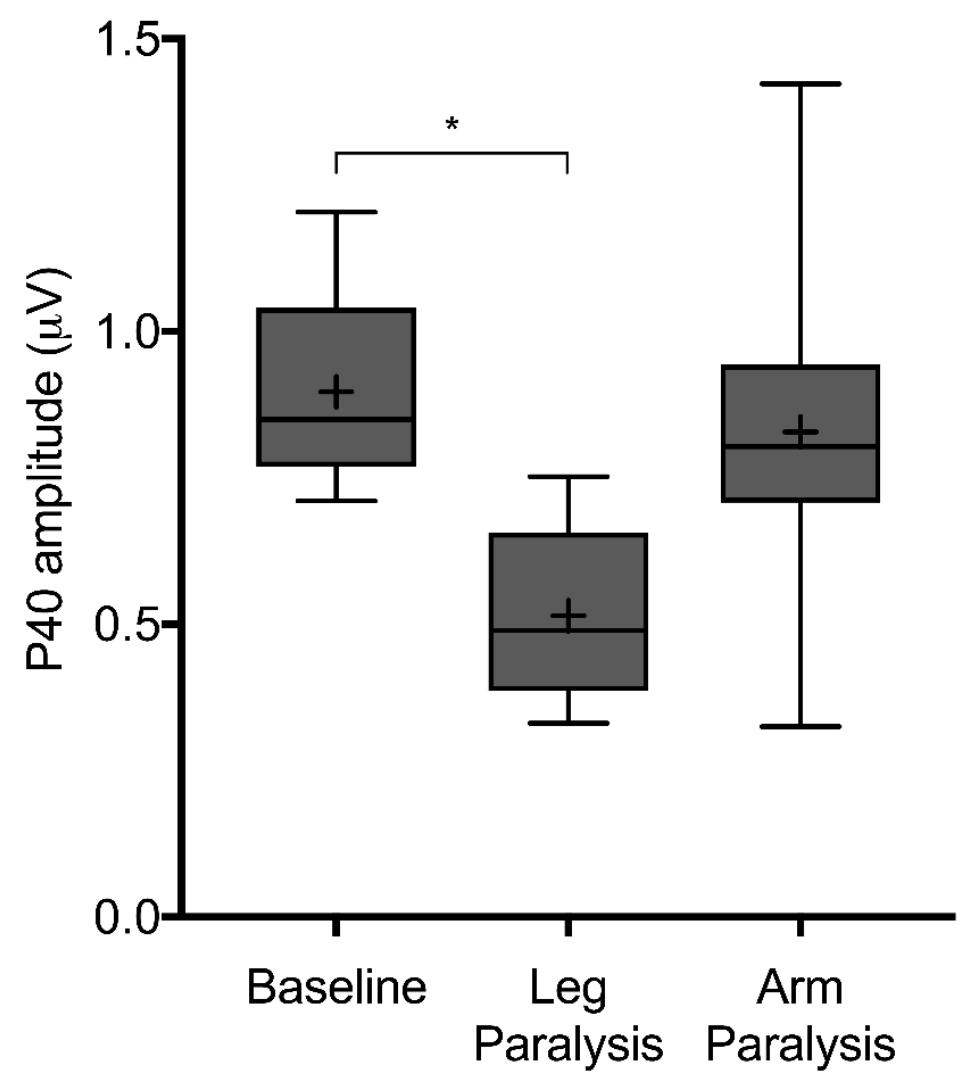

568 Figure 2. Mean amplitude $(\mu \mathrm{V})$ of $\mathrm{CPz}$ for the $\mathrm{P} 40$ component across experimental 569 conditions. Asterisk $(*)$ denotes significant differences $(p<0.05)$.

\section{0}



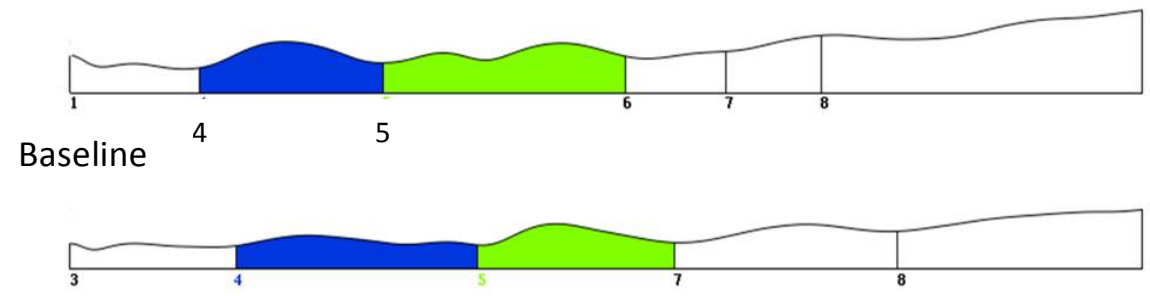

Leg paralysis

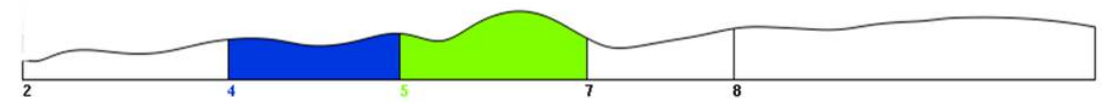

Arm paralysis

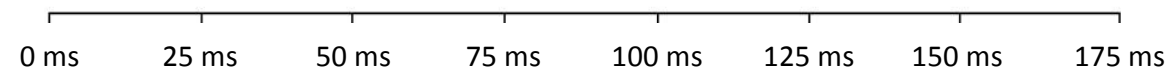

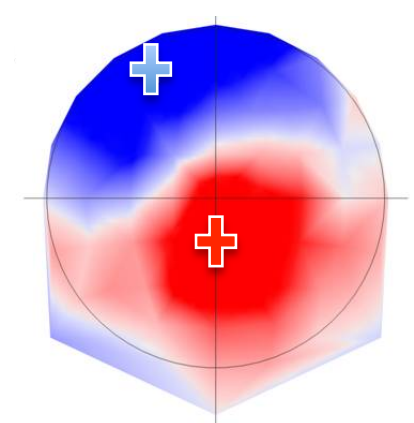

Map 4

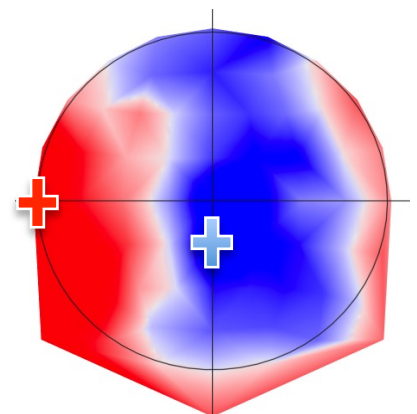

Map 5
574

575

576

577

578

579

580

581

582

Figure 3. Segments of stable brain topography (GFP) across all three experimental conditions. Maps 4 (blue) and 5 (green) denote the topography that corresponds to the SEP window. Topographical polarity for Map 4 (on the left) and Map 5 (on the right) is displayed in. The blue cross denotes the area of maximum negativity and the red cross denotes the area of maximum positivity. 


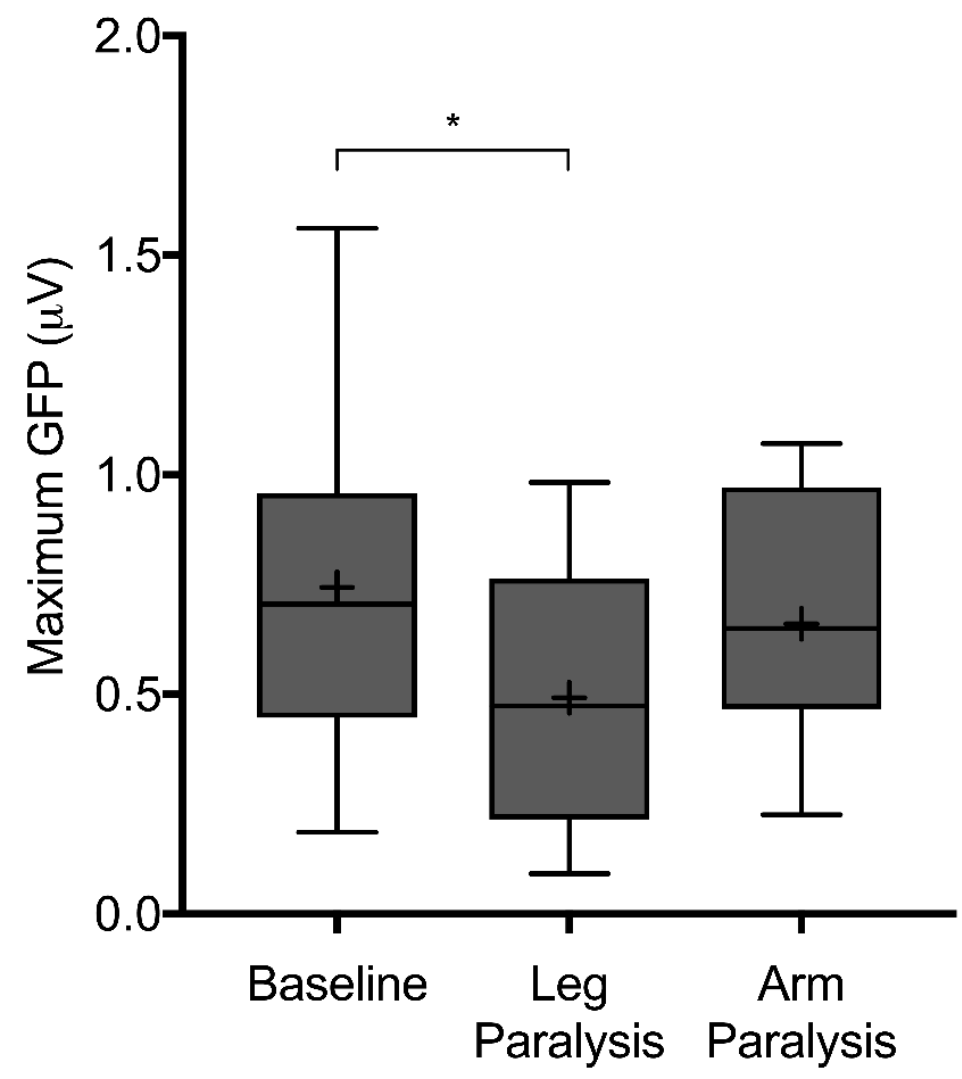

587 Figure 4. Maximum GFP was significantly lower during the Imagined Leg Paralysis

588

condition for Map 4 as compared to Baseline. Asterisk $\left(^{*}\right)$ denotes significant differences $(p$ $589<0.05)$.

590

591 


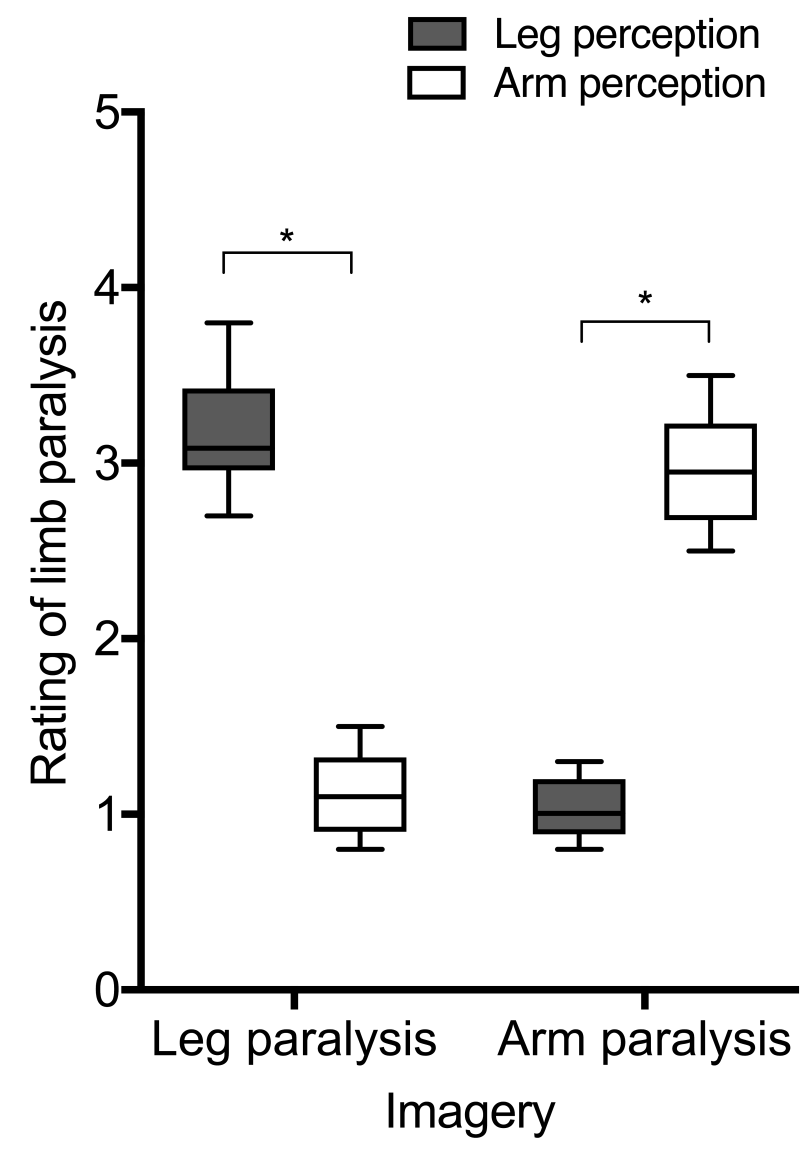

593

594 Figure 5. Box-and-whiskers plots of the perception of either the legs or arms during paralysis 595 imagery. Asterisk $(*)$ denotes significant differences $(p<0.05)$.

596

597 


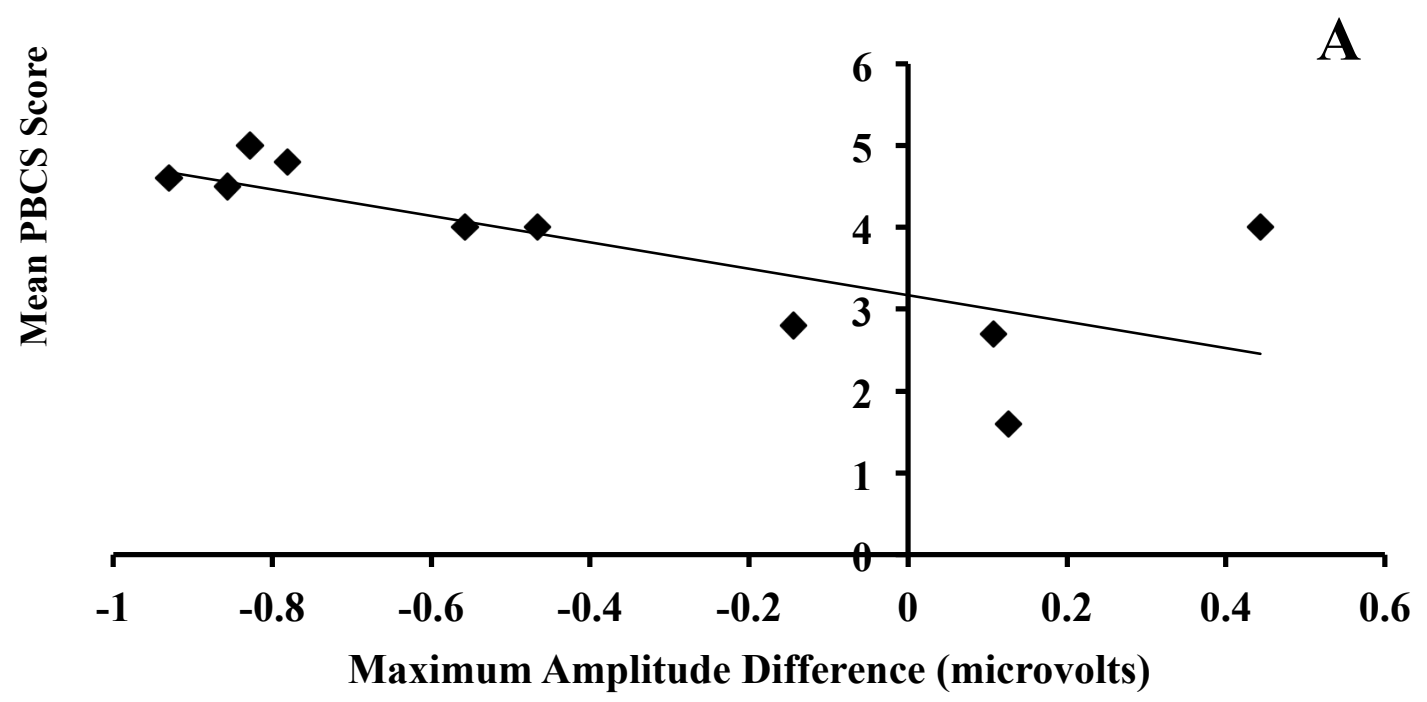

599

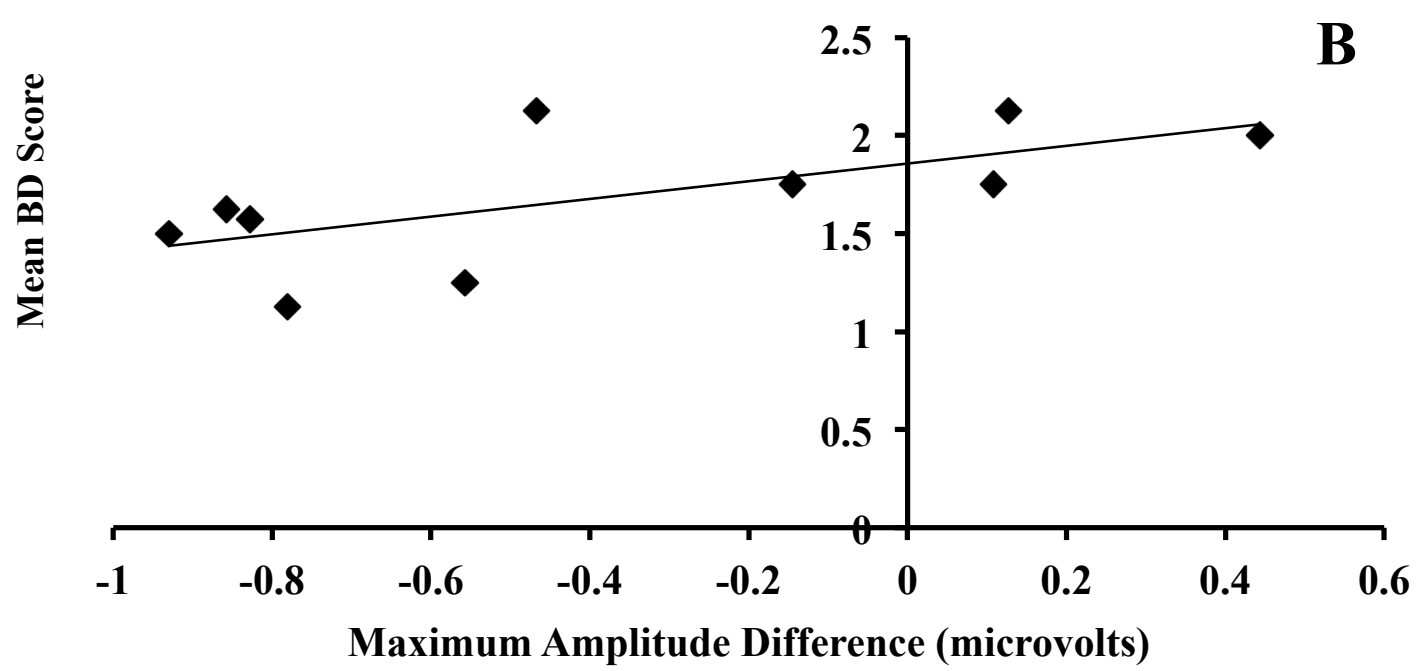

600

601 Figure 6. Plots show the maximum peak amplitude difference between the Imagined Leg 602 Paralysis and the Baseline conditions relative to the mean score of the PBCS (A) and BD 603 subscale (B). Analysis revealed a significant negative correlation between the amplitude 604 difference with PBSC scores $(\rho=-0.78, p=0.008)$ and a significant positive correlation 605 between the amplitude difference and BD scores $(\rho=0.65, p=0.028)$. 